\title{
Morphometrics for the Life Sciences
}




\section{Recent Advances in Human Biology}

Series Editor: Charles E. Oxnard (The University of Westem Australia)

\section{Published}

Vol. 1: The Origin \& Past of Modern Humans as Viewed from DNA eds. S. Brenner \& K. Hanihara

Vol. 2: Bone Structure and Remodeling ods. A. Odgaard \& H. Weinans

Vol. 3: The Origins and Past of Modem Humans - Towards Reconciliation eds. Philip V. Tobias \& Keiichi Omoto

Vol. 4: The Natural History of the Doucs and Snub-nosed Monkeys N. G. Jablouski

Forthcoming

Vol. 5: Bone \& Bones in Human Evolution: The Interaction of Architectures, Activity \& Adaptation

C. E. Oxnard

Vol. 6: Bone \& Fossils in Human Evolution: The Interaction of Morphology, Molecules \& Measurement C. E. Oxnard 
Series editor: Charles E. Oxnard

Centre for Human Biology

The University of Western Australia

\section{Morphometrics for the Life Sciences}

\section{Pete E. Lestrel}

Universiry of Colifornio, Los Angeles 


\section{Published by}

World Scientific Publishing Co. Pte. Ltd.

P O Box 128, Farrer Road, Singapore 912805

USA office: Suite 1B, 1060 Main Street, River Edge, NJ 07661

UK office: 57 Shelton Stroet, Covent Garden, London WC2H 9HE

\section{Britiah Library Cataloguing-in-Publication Data}

A catalogue record for this book is available from the British Library.

\section{MORPHOMETRICS FOR THE LIFE SCIENCES}

Copyright 02000 by World Scientific Publishing Co. Pte. Ltd.

All rights reserved. This book, or parts thereof, may not be reproduced in any form or by any means, electronic or mechanical, including photocopying, recording or any information storage and retrieval system now known or to be invented, without written permission from the Publisher.

For photocopying of material in this volume, please pay a copying fee through the Copyright Clearance Center, Inc., 222 Rosewood Drive, Danvers, MA 01923, USA. In this case permission to photocopy is not required from the publisher.

ISBN 981-02-3610-7

Printed in Singapore. 


\section{PROLOGUE}

One aim of this volume is to introduce the subject matter of a comparatively new discipline, but one with ancient roots: morphometrics. Morphometrics is the quantitative description or measurement of the biological form. It represents the initial step in the elucidation of taxonomic and classification questions, in dealing with issues of structure and function, as well as providing the raw material for explanations of biological process. Thus, morphometrics is central to all the biological sciences. Morphometrics deals with the need to quantify, in a complete and precise manner, the visual information inherent in all biological organisms. While this visual information is readily apparent, it has been inexorably difficult to adequately characterize in numerical terms.

As a starting point for the study of morphometrics, when measurement is considered, it is the universally used conventional metrical approach (CMA), consisting of distances, angles and ratios that are immediately thought of. That CMA represents a very inefficient procedure for dealing with the complex and irregular forms that constitute a majority of biological organisms on earth is generally not recognized. Accordingly, a re-evaluation of the way such metrics continue to be used is imperative and has spawned a number of alternative methods over the last three decades. While a complete morphometric description of biological organisms is not yet possible, the appearance of these new approaches can be considered a promising endeavor for continuing advances in morphometrics as the 21 st century progresses.

Another aim of this work is to continue in a more generalized setting, the application of Fourier Descriptors (FDs), Fourier Transforms (FTs) and wavelets to characterize the boundary outline of biological forms, which is taken up in Chapter 9. Part of this material represents: [1] a continuation of earlier research efforts and [2] an expansion of ideas initially formulated in an edited volume entitled: Fourier Descriptors and their Applications in Biology (1997).

Part one, Theoretical Background, consisting of six chapters is intended as an introduction to morphometrics. Chapters 1 and 2 introduce morphometrics and research methods respectively. These are followed by Chapter 3, which contains a brief history of measurement, which leads into Chapter 4, which describes the increasing use of quantification in biology. The last two chapters are more theoretical in nature and deal with a host of issues that impinge directly and indirectly on the quantitative analysis of the biological form. These include topics such as complexity, systems and models (Chapter 5) and the development of a formal model of form (Chapter 6).

Part two, Morphometric Techniques, is composed of four chapters followed by an epilogue. These chapters are more applied in character and deal with the numerous and quite diverse morphometric procedures that have been applied to biological datasets. Chapter 7 deals with multivariate morphometrics, Chapter 8 with coordinate 
morphometrics, Chapter 9 with boundary morphometrics and Chapter 10 with structural morphometrics. A number of sources are provided that allow access to available software that can be used with the methods described in Chapter 7 through Chapter 10. Additionally, more sources are available via the Internet and the student will find that a diligent search will be rewarded.

Most of the material in this volume lends itself for a one-semester interdisciplinary course on morphometrics. It is presented at an introductory level, although extensive references are provided for the inquisitive student who wants to probe more deeply. While it is aimed at the biologically oriented student, it may also be profitably used by students in other related disciplines. An introductory course on morphometrics has been given to post-graduate students at the School of Dentistry, University of California at Los Angeles.

Various parts of the text have been presented at lectures given to the faculty and students at the University of Glasgow Dental School; University of Adelaide, Australia; The University of Otago, Dunedin, New Zealand; The Karolinska Institute, Stockholm; Tokyo Metropolitan University, Hachioji, Tokyo; Tokyo National Science Museum, Ueno, Tokyo; Nihon University School of Dentistry at Matsudo, Chiba; The National University of Singapore; and the University of Bourgogne, Dijon.

Pete E. Lestrel

Van Nuys, California

February 2000 


\section{CONTENTS}

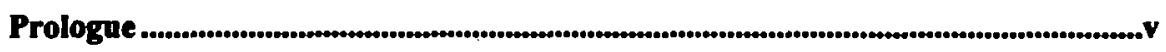

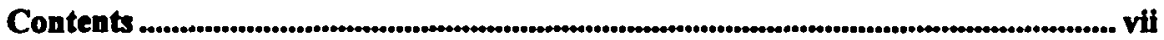

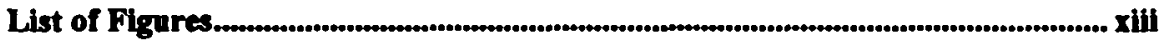

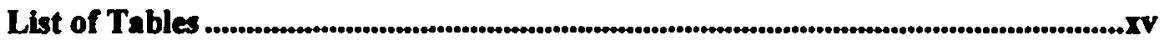

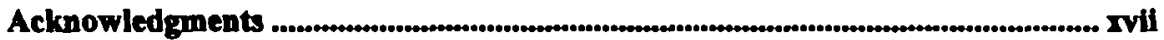

PART ONE: THEORETICAL BACKGROUND

1. INTRODUCTION TO MORPHOMETRICS.

1.1. INTRODUCTION ................................................................................... 1

1.1.1. The Visual Process............................................................................ 2

1.1.2. A Dual View of the World ..................................................................... 3

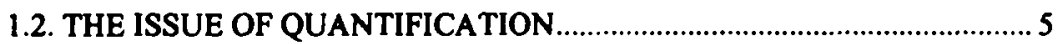

1.2.1. What is Morphometrics? ................................................................ 7

1.2.2. From Morphology to Process................................................................ 9

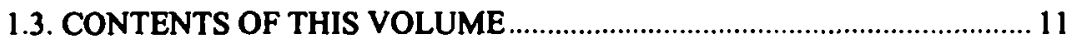

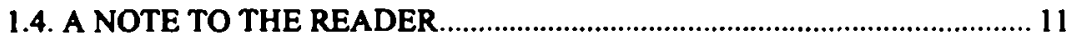

KEY POINTS OF THE CHAPTER ............................................................... 13

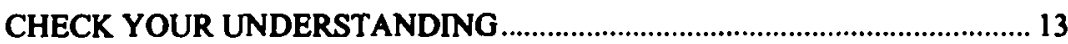

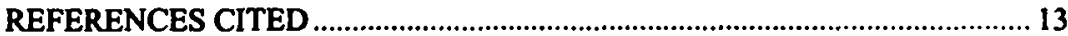

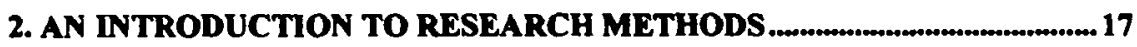

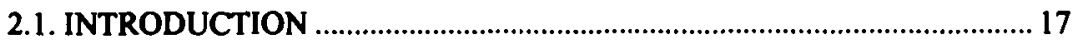

2.1.1. Definitions of Science .................................................................. 18

2.1.2. The Scientific Method ............................................................................. 19

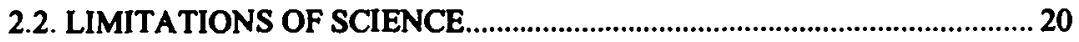

2.2.1. Ethical Considerations ........................................................................ 20

2.2.2. Principle of Independence ................................................................. 22

2.3. SOME STATISTICAL CONSIDERATIONS ………….............................. 23

2.3.1. Bias toward the Use of Statistics.......................................................... 24 
2.3.2. Types of Research Studies ............................................................... 24

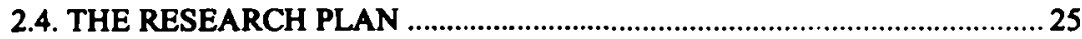

2.4.1. Initial Steps.................................................................................. 25

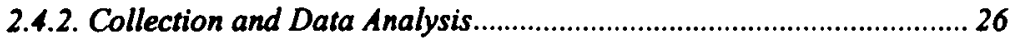

2.4.3. Some Other Requirements ........................................................... 27

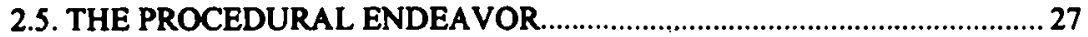

2.5.1. From the Literature Search to Hypotheses....................................... 28

2.5.2. The Research Design .................................................................... 32

2.5.3. Research Results ........................................................................ 35

2.6. THE DOCUMENTATION ENDEAVOR

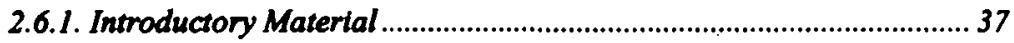

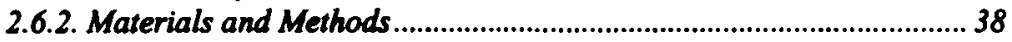

2.6.3. Results and Conclusions............................................................... 39

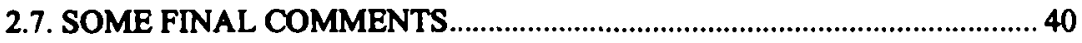

KEY POINTS OF THE CHAPTER...........................................................4 41

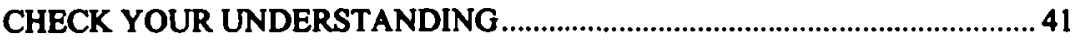

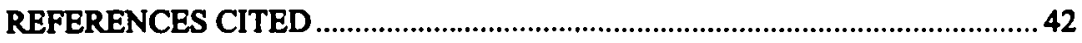

3. A HISTORY OF SCIENTIFIC MEASUREMENT

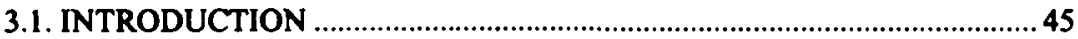

3.1.1. Precursors of Science ..................................................................46

3.1.2. Development of Language .......................................................47

3.2. EARLY BEGINNINGS OF MEASUREMENT ..................................... 48

3.2.J. The First Civilization: Mesopotamia .............................................. 50

3.2.2. Egyptian, Roman and Later Accomplishments......................................5I

3.2.3. Developments on the Indian Subcontinent ..................................... 52

3.2.4. Rise of Chinese Civilization.............................................................. 53

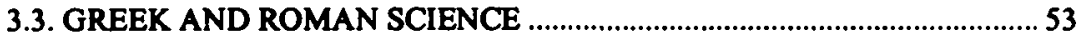

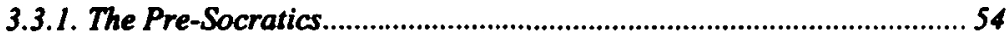

3.3.2. From Pythagoras to Democritus................................................... S5

3.3.3. Platonic and Aristotelian Philosophy.......................................... 58

3.3.4. Greco-Roman Achievements .....................................................62 62

3.4. THE HELLENISTIC PERIOD INTO MEDIEVALISM..........................65

3.4.1. The Islamic Contribution................................................................66 66

3.4.2. Early Medieval Philosophical Developments..................................68

3.4.3 The Ptolemaic Worldview..............................................................6 68

3.5. FROM THE RENAISSANCE TO THE ENLIGHTENMENT ....................69

3.5.1. The Copernican Revolution..........................................................69 69

3.5.2. Developments Leading to Newton and Beyond.............................. 75 


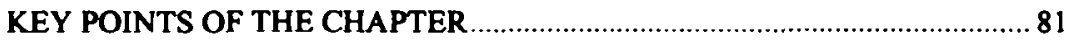

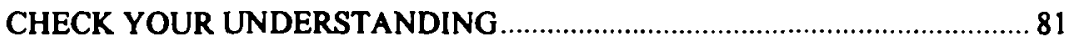

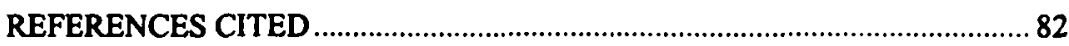

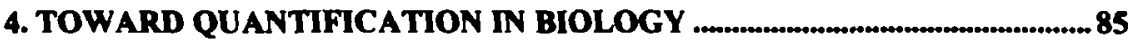

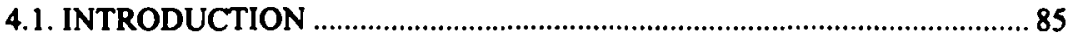

4.1.1. Classification of Organisms ..............................................................86

4.1.2. The Influence of Embryology on Heredity.......................................8 88

4.2. BEGINNINGS OF QUANTIFICATION IN BIOLOGY ...........................89

4.2.1. The Rise of Classical Genetics ....................................................... 90

4.2.2. The Roots of Statistical Theory ....................................................... 91

4.2.3. The Controversy over Biological Variation ................................... 92

4.2.4. The Evolutionary Synthesis ............................................................ 96

4.3. THE QUANTITATIVE STUDY OF FORM ..............................................99

4.3.1. Early Developments in the Study of Morphology........................... 100

4.3.2. From Morphology to Morphometrics............................................ 10I

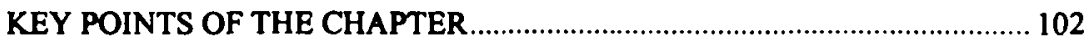

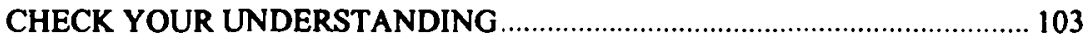

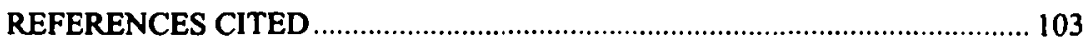

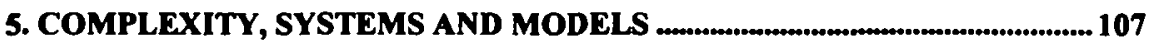

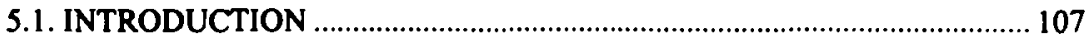

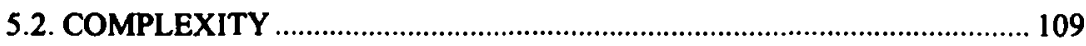

5.2.1. Complex Adaptive Systems .......................................................111

5.2.2. Properties of Emergence and Self-Organization ........................... 114

5.2.3. Morphogenesis and Complexity.................................................... 116

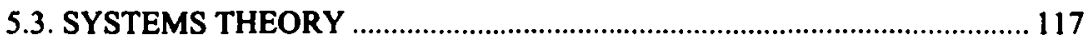

5.3.1. Systems Applications in Biology ................................................... 118

5.3.2. The Systems Approach ................................................................ 118

5.4. THE DEVELOPMENT OF MODELS..................................................... 119

5.4.1. Model Building.............................................................................. 120

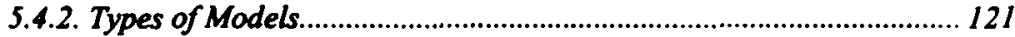

5.4.3. Modeling: A Simple Example ....................................................... 122

5.4.4. Other Approaches to Modeling .................................................. 126

5.4.5. The Challenge: Modeling the Development of Form ........................ 128

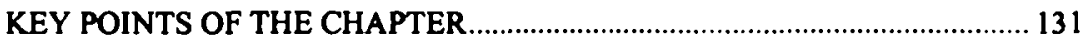

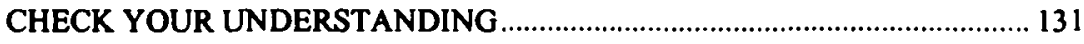

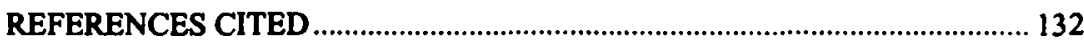




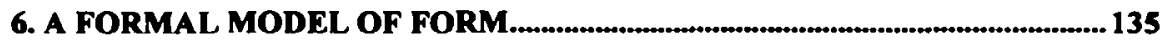

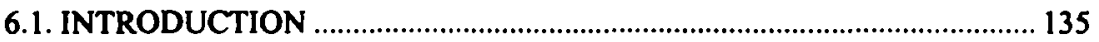

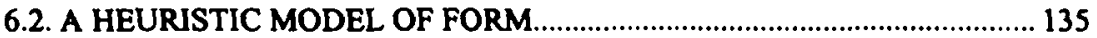

6.2.1. Justification for Quantilative Models of Form.................................. 135

6.2.2. Developing a Concept of Form ............................................................. 136

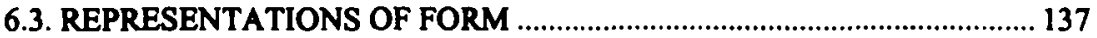

6.3.1. Specific Properties of Form............................................................... 137

6.3.2. Size, Shape and Structural Considerations .......................................... 139

6.3.3. A More Realistic Model of Form...................................................... 141

6.3.4. A Dynamic Model of Form ................................................................... 143

6.4. MORPHOMETRIC PROCEDURES........................................................ 146

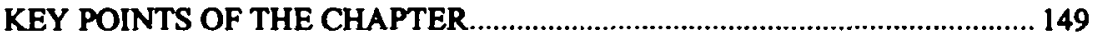

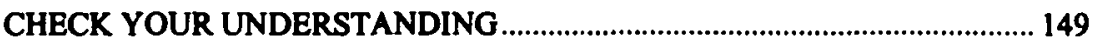

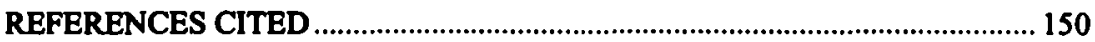

\section{PART TWO: MORPHOMETRIC TECHNIQUES}

7. MULTIVARIATE MORPHOMETRICS._. 155

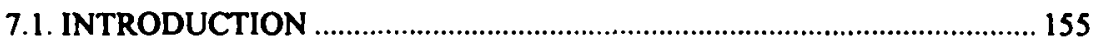

7.1.1. Historical Background ......................................................................... 155

7.1.2. Multivariate Procedures..................................................................... 156

7.1.3. Eigenvalues and Eigenvectors............................................................. 158

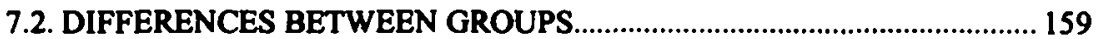

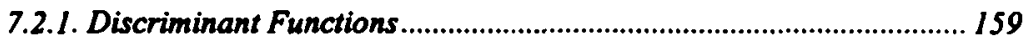

7.2.2. Mahalanobis $D^{2}$ Statistic............................................................. 160

7.2.3. Canonical Variate Anabsis............................................................. 161

7.2.4. Cluster Analysis .................................................................................... 164

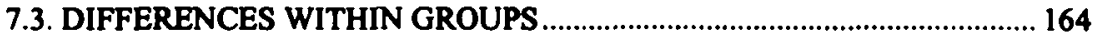

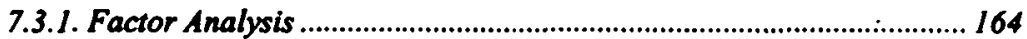

7.3.2. Principal Components Analysis ........................................................... 166

7.4. SOME FINAL COMMENTS....................................................................... 167

KEY POINTS OF THE CHAPTER.................................................................... 167

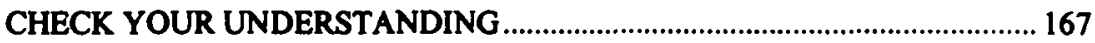

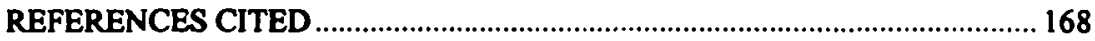

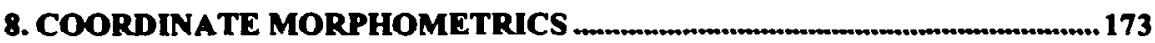

8.1. INTRODUCTION .................................................................................... 173

8.2. THE CONVENTIONAL METRICAL APPROACH .................................... 173

8.2.1. The Issue of Point Homology ........................................................... 173 
8.2.2. The Conventional Method (CMA)............................................. 175

8.2.3. Use of Angles and Ratios as Shape Measures................................. 175

8.2.4. Some Other Deficiencies with CMA ............................................. 178

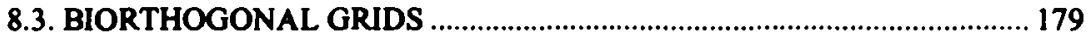

8.3.1. Basis for Biorthogonal Grids ......................................................... 179

8.3.2. Constraints of Biorthogonal Grids ............................................. 180

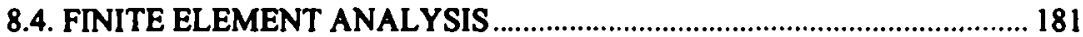

8.4.1. Finite Elements in 2-D............................................................. 182

8.4.2. Finite Elements in 3-D............................................................... 183

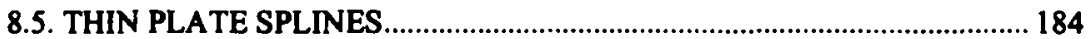

8.5.I. The Interpolation Function .......................................................... 184

8.5.2. Visualization of the Thin Plate Spline............................................. 184

8.6. EUCLIDEAN DISTANCE MATRIX ANALYSIS .................................. 186

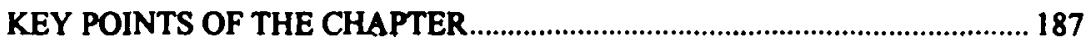

CHECK YOUR UNDERSTANDING ............................................................ 187

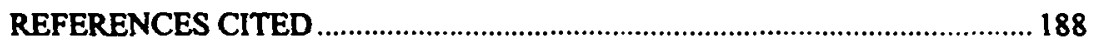

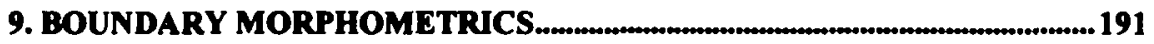

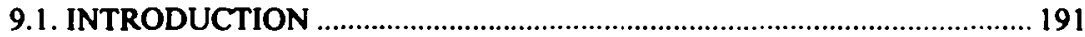

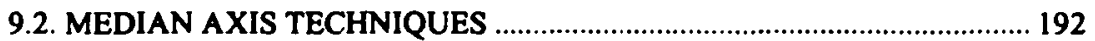

9.2.1. Definition of the Median Axis.................................................. 192

9.2.2. Variations on a Theme................................................................. 193

9.3. CONVENTIONAL FOURIER DESCRIPTORS ....................................... 196

9.3.1. Frequency, Amplitude and Phase Relationships................................ 196

9.3.2. Fourier's Series ............................................................................ 197

9.3.3. Fourier's Series as Discrete Approximations................................... 198

9.3.4. Residuals, Positional-Orientation and Size-Standardization ............ 200

9.3.5. Applications Using FDs ............................................................ 202

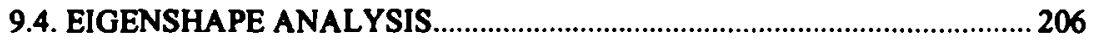

9.4.1. Algorithm for Eigenshape Analysis ................................................ 206

9.4.2. Procedures Involved in Eigenshape Analysis................................... 206

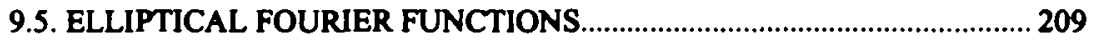

9.5.1. The Kuhl and Giardina Parametric Formulas............................... 210

9.5.2. Amplitude, Power and Phase Relationships................................... 21I

9.5.3. Other Elliptical Fourier Function Parameters ............................. 212

9.5.4. Positional-Orientation and Size-Standardization Revisited............. 212

9.5.5. Homology Once More................................................................... 212

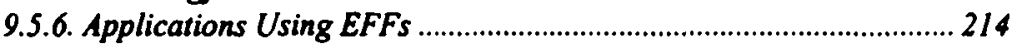

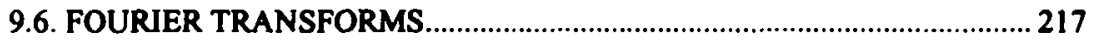


9.6.1 The Discrete Fourier Transform ..................................................... 217

9.6.2. The Fast Fourier Transform........................................................... 220

9.6.3. The Short Time Fourier Transform................................................221

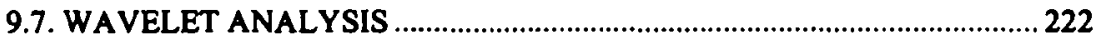

9.7.1. The Continuous Wavelet Transform ............................................. 222

9.7.2. The Discrete Wavelet Transform..................................................... 223

9.7.3. One-Dimensional Wavelet Applications........................................225

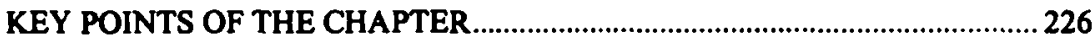

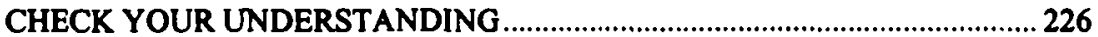

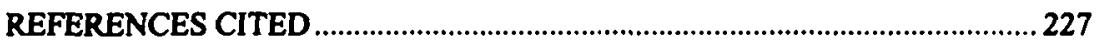

10. STRUCTURAL MORPHOMETRICS. 235

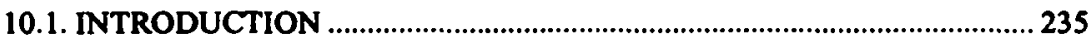

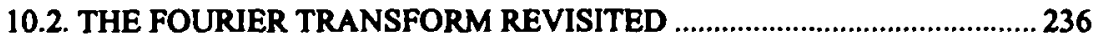

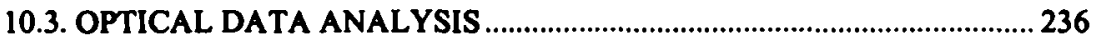

10.3.1. The One-Dimensional Optical Power Spectrum.............................. 236

10.3.2. The Two-Dimensional Optical Power Spectrum............................. 237

10.3.3. Two-Dimensional Optical FT Applications .................................. 238

10.4. TWO-DIMENSIONAL WA VELETS..................................................... 240

10.4.1. Wavelet Analysis of Two-Dimensional Images .............................. 240

10.4.2. Two-Dimensional Wavelet Applications ...................................... 242

KEY POINTS OF THE CHAPTER............................................................... 244

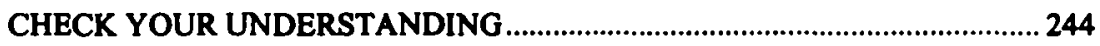

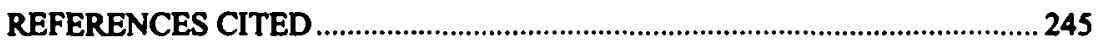

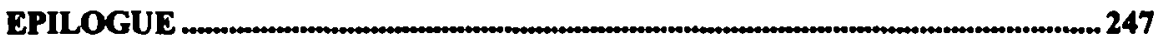

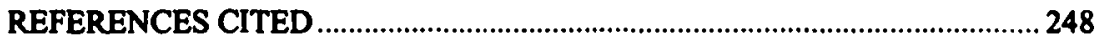

APPENDIX I. EFF23: A COMPUTER PROGRAM 249

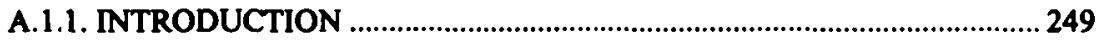

A.1.2. BRIEF OVERVIEW OF EFF23 …...................................................2 249

APPENDIX II. EFF23 PROGRAM FLOWCHARTS

INDEX 


\section{LIST OF FIGURES}

Figure 1.1. Modeling of biological processes................................................................ 10

Figure 5.1. The Newtonian system................................................................................ 117

Figure 5.2. A simple model of fish population dynamics using STELLA ................... 125

Figure 5.3. The logistic curve derived from the model using STELLA .......................... 126

Figure 5.4. A potential model of form changes during growth...................................... 130

Figure 6.1. Some morphological attributes of form....................................................... 141

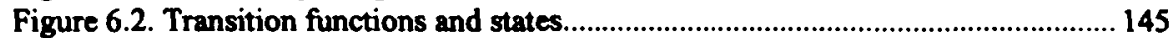

Figure 6.3. A procedural approach to modeling. …….................................................. 146

Figure 6.4. A schematic flowchart of morphometric procedures. ................................. 149

Figure 7.1. Mandibular superimpositions of normal human dentitions. ........................ 162

Figure 7.2. Mandibular superimpositions of crowded human dentitions...................... 162

Figure 7.3. A canonical plot of the mandibular data. ................................................ 163

Figure 8.1. The conventional metrical approach......................................................176

Figure 8.2. Form variation in the occipital aspect. .................................................... 177

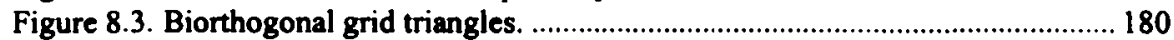

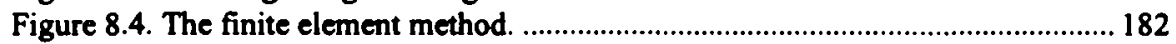

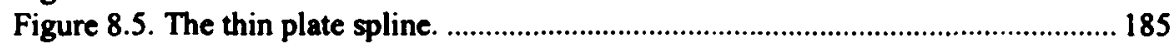

Figure 9.1. Median axis applied to a human mandible............................................. 193

Figure 9.2. Cranial vault of $H$. sapiens and $H$. neanderthalensis (harmonics 1 and 3). 203

Figure 9.3. Cranial vault of $H$. sapiens and $H$. neanderthalensis (harmonics 2 and 4). 203

Figure 9.4. Stepwise fit of the EFF to the mandibular morphology............................. 215

Figure 9.5. The Bounded outlines of the rabbit orbit in 3-D.......................................217

Figure 10.1. Optical Fourier transforms of the lumbar vertebrae. .................................. 239

Figure 10.2. Image of a horse and its associated 2-D wavelet decomposition. ............. 241

Figure 10.3. Compression of fingerprint data using wavelets. ...................................... 242

Figure A.1. Flowchart of data derived from EFF analysis.............................................2.250

Figure A.2. Computation of amplitude, power and phase............................................. 251

Figure A.3. Additional data extractable from EFFs........................................................... 251 
This page is intentionally left blank 


\section{LST OF TABLES}

Table 2.1. The research protocol................................................................................. 25

Table 2.2. Plan of research procedures......................................................................... 28

Table 2.3. Attributes of hypotheses. ............................................................................32

Table 2.4. Writing up the research results....................................................................... 36

Table 5.1. Some aspects of complexity ................................................................... 110

Table 5.2. Mechanisms of complexity......................................................................113

Table 5.3. The systems approach............................................................................. 119

Table 5.4. The modeling process..................................................................................... 121

Table 6.1. Some properties of form representations.................................................... 138

Table 6.2. Coordinate transformations. ........................................................................... 139

Table 6.3. Attributes of a more realistic model of form.............................................. 142

Table 6.4. The four major divisions of morphometrics.................................................. 147

Table 8.1. Measures of occipital form using indices. ....................................................... 177

Table 9.1. Steps involved in eigenshape analysis........................................................207

Table 9.2. Advantages and limitations of Fourier descriptors. .......................................213 
This page is intentionally left blank 


\section{ACKNOWLEDGMENTS}

The idea for this volume was in the back of my mind even before I had completed Fourier Descriptors and their Applications in Biology for Cambridge Press. However, its birth, so to speak, was a chance encounter, a consequence of my visiting Professor Charles Oxnard in Perth, Australia in 1996. Charles suggested I should consider writing it as a volume for the Recent Advances in Human Biology series of which he was Editor. Thus, Morphometrics for the Life Sciences was born. I am, needless to say, exceedingly grateful to Charles for that suggestion.

I am most indebted to Professor Fumio Ohtsuki, Tokyo Metropolitan University (TMU) for always being a gracious host starting with my first visit to Tokyo in 1995. In addition, for allowing me time in August of 1996 to initiate the first rough draft of the first two chapters of this volume, as well as having me return as Visiting Professor for three months in the summer of 1999 to supervise students and continue our joint research projects on human cranium shape changes and human growth in stature. I also wish to acknowledge the friendship of Professors Hideyuki Tanaka and Teruo Uetake, in whose company I was able to finally climb Mt. Fuji. I also want to mention my relationship with Dr. Osamu Takahashi, Nihon University School of Dentistry at Matsudo, who visited me for 15 months (1997-1999) to carry out a series of joint research projects dealing with human craniofacial complex. Finally, I want to indicate that I feel particularly honored by the gracious treatment I received from Dr. Shigeo Otake, Dean of the School, Professor Eisaku Kanasawa and Dr. Kazutaka Kasai, all from the Nihon University School of Dentistry where I delivered an invited lecture in June 1999.

I would like to thank Professor W. J. S. Kerr, University of Glasgow, Department of Orthodontics, for inviting me to beautiful Scotland and to participate in joint research on functional appliance therapy. Thanks must also go to Dr. Jan Huggare, Chairman of Orthodontics, University of Karolinska, for inviting me to come to Stockholm to give a lecture as well as allowing me the privilege to attend the Nobel Lecture in Physiology/Medicine for 1996 given to Professors Peter C. Doherty and Rolf M. Zinkernagel for their work concerning the specificity of cell-mediated immune defenses. Moreover, Jan was also instrumental in arraigning my 1999 lecture at the National University of Singapore when he was there for a year's appointment.

I am most grateful to Professor Eric Verrecchia, University of Bourgogne, Dijon, for inviting me to the Bio Geo Images ' 99 conference held in September 1999. Eric and I not only shared a fondness for the food and wine of the Burgundy region, but also a mutual admiration for the exceptional accomplishments of J. B. J. Fourier. I also wish to thank Dr. Hervé Drolon, University of Le Havre, for sending me reprints and for her helpful suggestions with respect to wavelets. 
xviii

A debt of gratitude goes to Professor Neal Garrett, UCLA School of Dentistry for reviewing parts of the manuscript. I have heavily relied on his advice in the past so I am again grateful for his efforts. I am also especially grateful to Charles Wolfe for his unflagging long-term programming efforts, valuable and stimulating discussions, and for the thorough review of the manuscript. Thanks must go to Dr. Albert Bodt for taking the time out of a busy medical practice at Kaiser Permanente, to review the manuscript; and for his enthusiasm and close friendship for almost twenty years now. Any errors remaining, however, are solely my own.

Lastly and most certainly not least, I owe my wife Dagmar a great deal, not only whose talents as a librarian were particularly helpful, but also for being my soul mate in marriage for the last thirty-two years and who had to tolerate my many late nights and early mornings necessary to bring this project to completion. 